\title{
Streptococcus mutans biofilm formation: utilization of a gtfB promoter-green fluorescent protein (PgtfB::gfp) construct to monitor development
}

\author{
Akihiro Yoshida† and Howard K. Kuramitsu
}

Author for correspondence: Howard K. Kuramitsu. Tel: +1 716829 2068. Fax: +1 7168293942. e-mail:kuramits@buffalo.edu

Department of Oral Biology, State University of New York, 3435 Main St, Buffalo NY 14214, USA
The glucosyltransferases of Streptococcus mutans are recognized as important virulence factors for this cariogenic bacterium. To study the expression of the gtfB gene of $S$. mutans in biofilms, a gtfB promoter (PgtfB)-green fluorescent protein (GFP) reporter system was developed. A Streptococcus-Escherichia coli shuttle vector harbouring a PgtfB:: gfp cassette was introduced into $S$. mutans GS-5, and the expression of GFP by the transformed S. mutans cells was confirmed by fluorescence microscopy. Furthermore, confocal laser scanning microscopy was carried out on biofilms attached to polystyrene plates; enhanced gtfB expression was observed in various microcolonies across these biofilms. To further test the hypothesis that gtfB expression is upregulated in biofilms, flow cytometry analysis was done on planktonic and biofilm cells; this analysis showed an approximately five-fold increase in gtfB expression in the biofilm cells relative to the planktonic cells. Real-time (TaqMan) PCR analysis confirmed that gtfB expression in the biofilm cells was enhanced relative to the planktonic cells. Previously, it has been suggested that the $S$. mutans gtfC gene might be co-transcribed with gtfB. Therefore, RT-PCR analysis was performed on gtfB-expressing $S$. mutans; this analysis demonstrated that gtfC was co-transcribed with gtfB. These results indicated that GFP expression can be utilized to examine gene regulation in S. mutans biofilm formation.

Keyword: glucosyltransferase (GTF)

\section{INTRODUCTION}

The extracellular polysaccharides synthesized from sucrose via the glucosyltransferases (GTFs) of Streptococcus mutans have been recognized to be important virulence factors for the induction of human dental caries (Loesche, 1986). S. mutans synthesizes $\alpha$ - $(1 \rightarrow 3)$ and $\alpha-(1 \rightarrow 6)$-linked glucan polymers through the concerted action of three secreted GTFs that are encoded by the genes $g t f B, g t f C$ and $g t f D$. The tandemly arranged $g t f B$ and gtfC genes encode the enzymes (GTF-I and GTF-SI, respectively) responsible for the synthesis of water-insoluble, $\alpha$-( $1 \rightarrow 3)$-rich glucan (IG) (Aoki et al., 1986; Hanada \& Kuramitsu, 1988). gtfD encodes GTF-

†Present address: Department of Preventive Dentistry, Kyushu University Faculty of Dental Science, Fukuoka 812-8582, Japan.

Abbreviations: CDM, chemically defined medium; CLSM, confocal laser scanning microscopy; $\mathrm{Ct}$, threshold cycle; gfp and GFP, green fluorescent protein; gtf and GTF, glucosyltransferase; PgftB, gftB promoter.
$S$, which is responsible for the synthesis of $\alpha-(1 \rightarrow 6)$ linked, water-soluble glucan (SG) (Hanada \& Kuramitsu, 1989). The principal roles of the extracellular glucans of $S$. mutans are to facilitate the adhesion of the organism to and its accumulation on teeth, and to establish an extracellular polysaccharide matrix for the organism that may provide it with increased protection against mechanical host-clearance forces and which may afford it some protection against host immune defences. S. mutans cells attached to teeth as part of a biofilm (dental plaque) may exhibit a distinct mode of growth which differs from that of planktonic cells, and this distinct mode of growth can be characterized by an increased resistance to antibiotics as well as by differential gene expression (Watnick \& Kolter, 2000).

The green fluorescent protein (GFP) from the jellyfish Aequorea victoria has been used as a visual marker of gene expression in prokaryotic and eukaryotic organisms. GFP has the advantage of being more sensitive 
than other reporter genes and requires no extraneous substrates or co-factors for its detection (Chalfie et al., 1994). Furthermore, mutants of GFP with increased fluorescence intensities have been constructed; for example, one GFP mutant, $g f p-m u t 2$, contains a triple substitution - S65A, V68L and S72A - and exhibits 100fold more fluorescence in Escherichia coli than the wildtype protein (Cormack et al., 1996). GFP has also been used as a species-specific marker to label adherent bacteria in biofilms (Skillman et al., 1998). Furthermore, GFP can be used to analyse the spatial distribution of bacteria in biofilms. Oral biofilms, in the form of dental plaque, are particularly complex because they consist of hundreds of bacterial species (Davey \& O'Toole, 2000); hence, the utilization of GFP to examine these communities should provide further insights into the spatial distribution of organisms within dental plaque.

Previously, molecular genetic and biochemical studies of the gtf genes have been carried out using planktonic $S$. mutans. In vitro studies have indicated that $g t f B$ and $g t f C$ are essential for the sucrose-dependent attachment of $S$. mutans cells to hard surfaces (Aoki et al., 1986) but gtfD is dispensable (Hanada \& Kuramitsu, 1989). Nevertheless, all three genes appear to be required for induction of maximal dental caries in a rat model (Yamashita et al., 1993). In addition, by using a chloramphenicol reporter gene, it has been demonstrated that expression of the gtfB-gtfC operon of $S$. mutans appears to be induced when the organism colonizes solid surfaces in vitro (Hudson \& Curtiss, 1990). However, since $g t f C$ can be transcribed from its own promoter (Smorawinska \& Kuramitsu, 1995), it is not clear if these effects were a result of increased transcription from $g t f B, g t f C$ or both promoters. To test the hypothesis that the expression of $S$. mutans gtfB is upregulated in biofilms, we have developed a Streptococcus-E. coli shuttle vector system to quantitate and visualize the expression of this gene in biofilms using plasmid-encoded GFP. The results described here confirm that $g t f B$ expression is upregulated in biofilm cells and also indicate that the transcription of $g t f C$ can be initiated from the $g t f B$ and the $g t f C$ promoters.

\section{METHODS}

Bacterial strains and plasmids. All of the bacterial strains and plasmids used in this study are listed in Table 1. Strains of $S$. mutans were routinely subcultured and maintained in ToddHewitt broth (THB; Difco). Transformants of $S$. mutans were selected following their growth on THB agar plates supplemented with $10 \mu \mathrm{g}$ erythromycin $\mathrm{ml}^{-1}$ or $500 \mu \mathrm{g}$ kanamycin $\mathrm{ml}^{-1}$.

DNA manipulations. DNA isolation, restriction digests, ligations and transformations of competent E. coli cells were carried out as described by Sambrook et al. (1989). Transformation of $S$. mutans was performed as described previously (Perry et al., 1983).

Construction of PgtfB::gfp fusion plasmids. The primers used in this study are listed in Table 2. Plasmid pKEN, containing gfp-mut2, was described by Cormack et al. (1996). The promoterless $g f p X b a \mathrm{I}-P s t \mathrm{I}$ cartridge was excised from $\mathrm{pKEN}$ and inserted into the XbaI-PstI sites of pUC19 to generate pAYG210. For amplification of the S. mutans GS-5 PgtfB region, oligonucleotides were synthesized based upon the nucleotide sequence of the promoter region of $g t f B$ including a portion of the $5^{\prime}$ region of $g t f B$ (GenBank accession no. M17361). To facilitate cloning of the PCR product into pAYG210, which contained the promoterless gfp-mut2 sequence, restriction sites were incorporated into the primers. The primer pair comprised gtfBF520(Bam) (complementary to nucleotides 520-537) and gtfBR712(Xba) (complementary to nucleotides 695-712). A PCR product was amplified from $S$. mutans GS-5 chromosomal DNA. The product was digested with $B a m \mathrm{HI}$ and $\mathrm{XbaI}$ and inserted in-frame into similarly digested pAYG210. A $g t f B:: g f p$ fusion fragment, digested with BamHI and PstI, from pAYBG310 was amplified by PCR using the primer pair gtfBF520(Sph)/gfpR(Sph) (Prasher et al., 1992). The resulting PCR product was digested with $S p h I$ and inserted into SphI-digested pDL276 (Dunny et al., 1991); the resulting plasmid was designated pAYBG754S. To introduce erythromycin resistance into pAYBG754S, the erythromycin cassette was amplified by PCR from pResEmMCS10 using primers AM1(Sma) and AM3(Sma), respectively (Brehm et al., 1987; Shiroza \& Kuramitsu, 1993). The resulting PCR product was digested with $S m a I$ and inserted into the SmaI site of pAYBG754S, to generate the erythromycin-resistant plasmid pAYBG854S. To generate a $g t f B:: g f p$ fusion by a Campbelllike insertion into the chromosome of $S$. mutans, a suicide plasmid harbouring the $g t f B:: g f p$ fusion was constructed. A $g t f B:: g f p$ fusion fragment, digested with BamHI and HindIII, from pAYBG310 was inserted into the BamHI- and HindIIIdigested integration plasmid pZ63 (Sato et al., 1991); the resulting plasmid was designated pAYBG554.

Fluorescence microscopy. Cultures of $S$. mutans strains were grown in chemically defined medium (CDM) supplemented with sucrose to an $\mathrm{OD}_{570}$ value of $0 \cdot 5$. The CDM contained $\left(1^{-1}\right) 2 \cdot 0 \mathrm{~g}$ L-glutamic acid, $0.2 \mathrm{~g}$ L-cysteine, $0.9 \mathrm{~g}$ L-leucine, $1.0 \mathrm{~g} \mathrm{NH}_{4} \mathrm{Cl}, 2.5 \mathrm{~g} \mathrm{~K}_{2} \mathrm{HPO}_{4}, 2.5 \mathrm{~g} \mathrm{KH}_{2} \mathrm{PO}_{4}, 4.0 \mathrm{~g} \mathrm{NaHCO}_{3}$, $1.2 \mathrm{~g} \mathrm{MgSO}_{4} .7 \mathrm{H}_{2} \mathrm{O}, 0.02 \mathrm{~g} \mathrm{MnCl}_{2} .4 \mathrm{H}_{2} \mathrm{O}, 0.02 \mathrm{~g} \mathrm{FeSO}_{4} .7 \mathrm{H}_{2} \mathrm{O}$, $0.6 \mathrm{~g}$ sodium pyruvate, $1.0 \mathrm{mg}$ riboflavin, $0.5 \mathrm{mg}$ thiamin $/ \mathrm{HCl}$, $0.1 \mathrm{mg}$ D-biotin, $1.0 \mathrm{mg}$ nicotinic acid, $0.1 \mathrm{mg} p$-aminobenzoic acid, $0.5 \mathrm{mg}$ calcium pantothenate, $1.0 \mathrm{mg}$ pyridoxal $/ \mathrm{HCl}$ and $0 \cdot 1 \mathrm{mg}$ folic acid, and was adjusted to $\mathrm{pH} 7 \cdot 0$ with $\mathrm{H}_{3} \mathrm{PO}_{4}$. To maximize $g f p$ expression, $1.0 \mathrm{M}$ phosphate buffer was added to the cultures to a final concentration of $50 \mathrm{mM}(\mathrm{pH} 7 \cdot 0)$ about $10 \mathrm{~min}$ before the microscopic analysis was done. The cell cultures were analysed directly by using phase-contrast fluorescent microscopy.

Flow cytometry analysis. gtfB expression by cells of $S$. mutans $854 \mathrm{~S}$ and GS-5 in their planktonic and biofilm forms was monitored by flow cytometry using an EPICS XL apparatus with XL SYSTEM II software (Beckman Coulter). Green fluorescence ( $\lambda_{\text {excitation }} 488 \mathrm{~nm}$; $\lambda_{\text {emission }} 515-545 \mathrm{~nm}$ ) was analysed. Dead cells were excluded from the analysis using forward- and side-scatter light gating. Planktonic and sessile cells for fluorescence-activated cell sorter (FACS) analysis were obtained following overnight anaerobic culturing of the strains in CDM supplemented with $0.5 \%$ sucrose in 6-well polystyrene plates (Corning). Under these conditions, the bottom surfaces of the wells were uniformly covered by the parental GS-5 cells. Briefly, after separating the planktonic and sessile cells, the sessile cells were washed several times with CDM. The sessile cells were then resuspended in fresh CDM and sonicated to disrupt the streptococcal chains, to avoid the effects of size differences. The planktonic cells were also sonicated, in a similar manner to the sessile cells. Both 
Table 1. Bacterial strains and plasmids used in this study

\begin{tabular}{|c|c|c|}
\hline Strain/plasmid & Relevant characteristics* & Source/reference \\
\hline S. mutans GS-5 & Wild-type; Ems & SUNYaB† \\
\hline S. mutans 554 & GS -5 containing chromosomally integrated pAYBG554; $\mathrm{Em}^{\mathrm{r}} \mathrm{Km}^{\mathrm{r}}$ & This study \\
\hline S. mutans $854 \mathrm{~S}$ & GS-5 containing pAYBG854S; $\mathrm{Em}^{\mathrm{r}} \mathrm{Km}^{\mathrm{r}}$ & This study \\
\hline E. coli $\mathrm{DH} 5 \alpha$ & Cloning host & Gibco-BRL \\
\hline \multicolumn{3}{|l|}{ Plasmids } \\
\hline pUC19 & Cloning vector; $\mathrm{Amp}^{\mathrm{r}}$ & New England Biolabs \\
\hline pKEN & $g f p-m u t 2 ; A m p^{r}$ & Cormack et al. (1996) \\
\hline pAYBG310 & pUC19 harbouring PgtfB::gfp; Amp ${ }^{\mathrm{r}}$ & This study \\
\hline pDL276 & Streptococcus-E. coli shuttle vector; $\mathrm{Km}^{\mathrm{r}}$ & Dunny et al. (1991) \\
\hline pZ63 & Streptococcus integration plasmid harbouring scrB::lacZ; $\mathrm{Em}^{\mathrm{r}} \mathrm{Km}^{\mathrm{r}}$ & Sato et al. (1991) \\
\hline pAYBG554 & pZ63 harbouring PgtfB::gfp instead of $s c r B:: l a c Z ; \mathrm{Em}^{\mathrm{r}} \mathrm{Km}^{\mathrm{r}}$ & This study \\
\hline pAYBG754S & pDL276 harbouring PgtfB::gfp; Km ${ }^{\mathrm{r}}$ & This study \\
\hline pResEmMCS10 & Streptococcus integration plasmid; $\mathrm{Em}^{\mathrm{r}} \mathrm{Amp}^{\mathrm{r}}$ & Shiroza \& Kuramitsu (1993) \\
\hline pAYBG854S & pDL276 harbouring PgtfB::gfp; $\mathrm{Em}^{\mathrm{r}} \mathrm{Km}^{\mathrm{r}}$ & This study \\
\hline
\end{tabular}

*Em ${ }^{\mathrm{s}}$, erythromycin-sensitive; $\mathrm{Em}^{\mathrm{r}}$, erythromycin-resistant; $\mathrm{Km}^{\mathrm{r}}$, kanamycin-resistant; $\mathrm{Amp}^{\mathrm{r}}$, ampicillin-resistant. † SUNYaB, Culture Collection of the Department of Oral Biology, State University of New York, Buffalo, NY, USA.

Table 2. Oligonucleotide primers and probes used in this study

\begin{tabular}{|c|c|c|}
\hline Oligonucleotide & Sequence* & Gene(s) \\
\hline \multicolumn{3}{|l|}{ Primer } \\
\hline gtfBF520(Bam) & 5'-CCC CAT CGA TAG ACT GTT GTT TGG TTG-3' & $\operatorname{PgtfB}$ \\
\hline gtfBR712(Xba) & 5'-CCC CTC TAG ACC ATT AGG AAC CTC CAA A-3' & $\operatorname{PgtfB}$ \\
\hline gtfBF520(Sph) & 5'-CCC CGC ATG CAG ACT GTT GTT TGG TTG-3' & PgtfB::gfp \\
\hline $\operatorname{gfpR}(\mathrm{Sph})$ & 5'-CCC CGC ATG CTT ATT TGT ATA GTT CAT C-3' & $P g t f B:: g f p$ \\
\hline AM1 (Sma) & 5'-CCC CCC GGG GAA GGA GTG ATT ACA TGA AC-3' & Erythromycin cassette \\
\hline $\mathrm{AM} 3(\mathrm{Sma})$ & 5'-CCC CCC GGG AGC GAC TCA TAG AAT TAT TTC-3' & Erythromycin cassette \\
\hline RT1F & 5'-TAC TTT AGA GCA AAC GGT-3' & $g t f B C$ \\
\hline RT1R & 5'-AGC AGT CAG AGT TTC TTT-3' & $g t f B C$ \\
\hline RT2F & 5'-CTT ATA CTG TTG GGG AAA-3' & $g t f B$ \\
\hline RT2R & 5'-GTA TCA TCA TAA GTC TTG-3' & $g t f B$ \\
\hline RT3F & 5'-ACA ACA AAC CAG TCT ACA-3' & $g t f C$ \\
\hline RT3R & 5'-CGG ACT GGT TGC TGT ATT-3' & $g t f C$ \\
\hline gtfB-F241 & 5'-CAT ACA GTA ACG ACA AGC AGT AGC TCT A-3' & $g t f B$ \\
\hline gtfB-R539 & 5'-GTA CGA ACT TTG CCG TTA TTG TCA TA-3' & $g t f B$ \\
\hline Sm16S-F1250 & 5'-CCG GTG ACG GCA AGC TAA-3' & $16 \mathrm{~S}$ rRNA \\
\hline Sm16S-R1319 & 5'-TCA TGG AGG CGA GTT GCA-3' & $16 \mathrm{~S}$ rRNA \\
\hline \multicolumn{3}{|l|}{ TaqMan probe } \\
\hline $\begin{array}{l}\text { GtfB TaqMan minor- } \\
\text { groove-binder }\end{array}$ & 5'-FAM-ATG TTA TTG ATG ATA GCA ATG C-NFQ-MGB-3' & $g t f B$ \\
\hline 16S rRNA TaqMan & 5'-FAM-CTC TGA AAG CCG ATC TCA GTT CGG ATT G-TAMRA-3' & $16 \mathrm{~S}$ rRNA \\
\hline
\end{tabular}

*Endonuclease recognition sequences are shown in bold. FAM, 6-carboxyfluorescein; NFQ, non-fluorescent quencher; MGB, minorgroove-binder; TAMRA, 6-carboxytetramethylrhodamine.

planktonic and sessile cells were added to $5 \mathrm{ml}$ of fresh CDM supplemented with $0.5 \%$ sucrose to an $\mathrm{OD}_{570}$ value of $0 \cdot 3$. Sodium phosphate buffer $(1.0 \mathrm{M})$ was then added to the suspensions to a final concentration of $50 \mathrm{mM}(\mathrm{pH} 7 \cdot 0)$ about 10 min before the FACS analysis was done.

Confocal laser scanning microscopy (CLSM). Microscopic observations and image acquisition were performed with a CLSM system using LSM 5 PASCAL with LSM 5 PASCAL software (Carl Zeiss); $\lambda_{\text {excitation }} 488 \mathrm{~nm}$ and $\lambda_{\text {emission }} 505-530 \mathrm{~nm}$ were analysed. Biofilms for CLSM analysis were prepared the same way as for the FACS analysis. Briefly, overnight cultures of $S$. mutans 854 S and GS-5 grown in CDM supplemented with $0.5 \%$ sucrose in 6 -well polystyrene plates (Corning) were washed several times with CDM and then fresh CDM was added to the wells. Water-immersion lenses (Plan-NEOFLUAR 25/0.81 mm; Carl Zeiss) were employed for this study. 


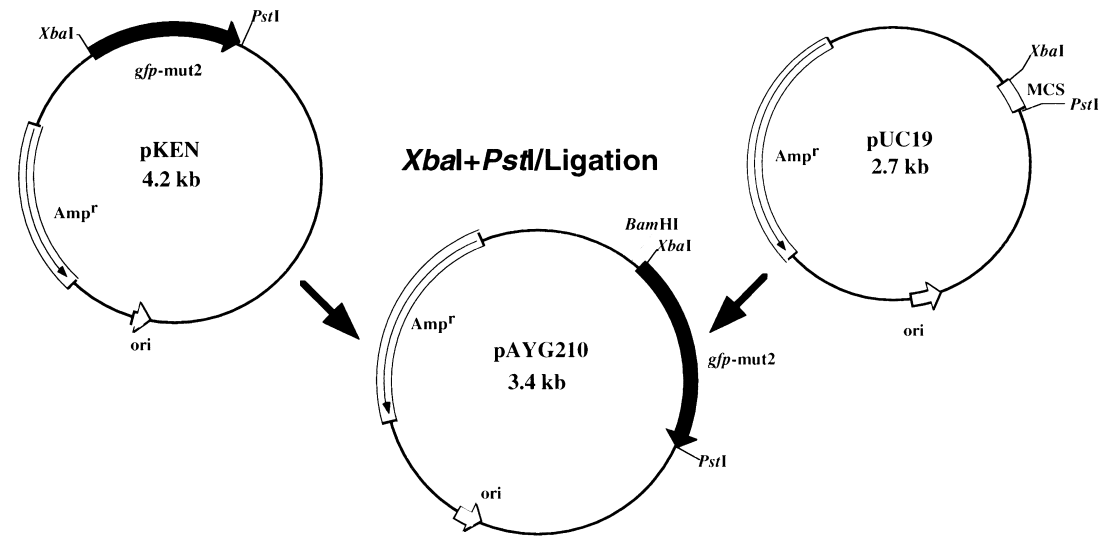

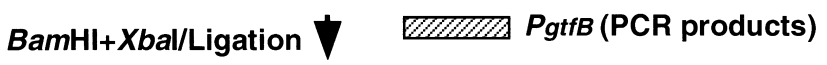

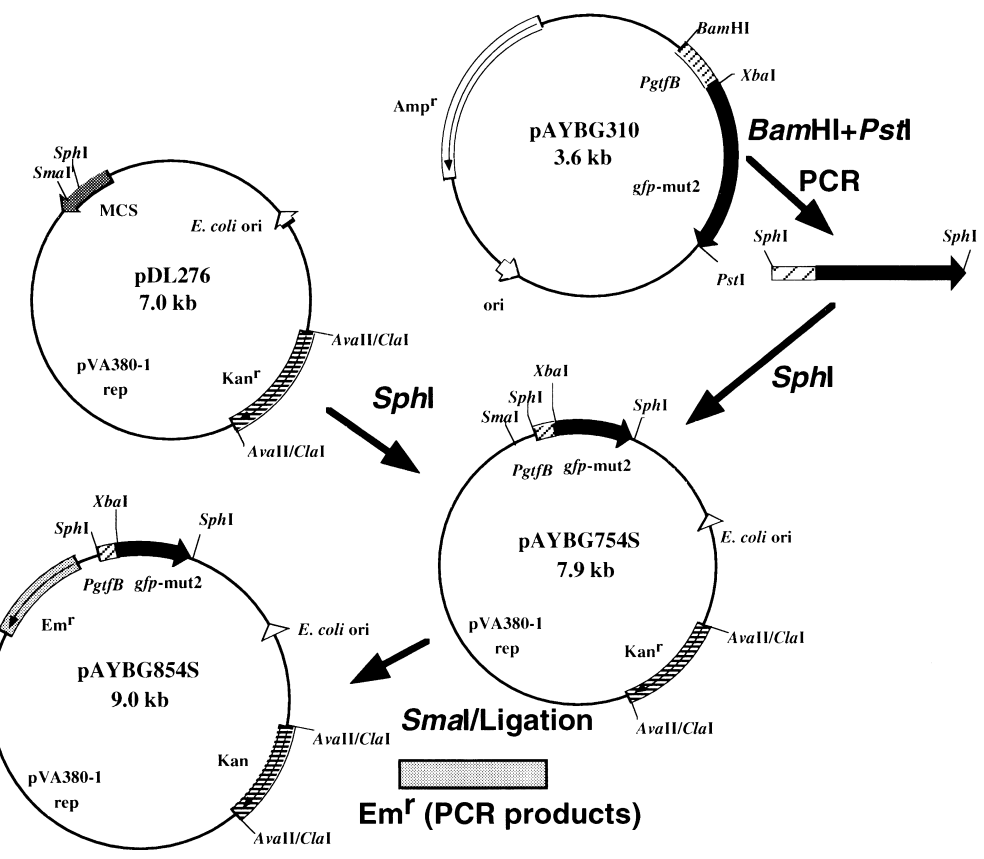

Fig. 1. Construction of the streptococcal gfp plasmid pAYBG854S. The promoterless gfp gene was subcloned into pUC19, resulting in pAYG210. A DNA fragment containing PgtfB was obtained by PCR amplification of S. mutans GS-5 chromosomal DNA and subcloned into the BamHI-Xbal site of pAYBG210 to generate pAYBG310. The gtfB::gfp fragment flanked by Sphl sites was amplified by PCR from pAYBG310 and subcloned into the Sphl site of pDL276 to generate pAYBG754S. An erythromycin cassette was then subcloned into the Smal site of pAYBG754S to generate pAYBG854S. MCS, multiple-cloning site; rep and ori, origin of replication.

RT-PCR analysis. Total RNA was isolated from $S$. mutans by using TRIZOL Reagent (Gibco-BRL), according to the manufacturer's instructions, and treated with RNase-free DNase (DNase I; Gibco-BRL). Reverse transcriptase reactions were performed by using SUPERSCRIPT II RNase $\mathrm{H}^{-}$Reverse Transcriptase (Gibco-BRL), according the manufacturer's instructions. For the RT-PCR experiment, primers were designed from the sequences of the $g t f B$ and gtfC genes; the primer pairs used are listed in Table 2. PCR amplifications were carried out with Taq polymerase (Promega) and the following cycling protocol: one cycle at $94^{\circ} \mathrm{C}$ for $5 \mathrm{~min}$ followed by 40 cycles at $94^{\circ} \mathrm{C}$ for $30 \mathrm{~s}, 47^{\circ} \mathrm{C}$ for $30 \mathrm{~s}$ and $72{ }^{\circ} \mathrm{C}$ for $1 \mathrm{~min}$. To check for DNA contamination, reverse transcriptase was not present in the negative control reaction.
Oligonucleotide primers and probes for real-time RT-PCR. Oligonucleotide primers and probes for $g t f B$ and $16 \mathrm{~S}$ rRNA (a housekeeping gene used as an internal control) were designed by using Primer Express 1.5 software (PE Biosystems). Fluorogenic probes were used to continuously monitor PCR product formation during PCR. The oligonucleotide probe for 16S rRNA was labelled with a reporter dye (6-carboxyfluorescein) covalently attached at its $5^{\prime}$ end and a quencher dye (6-carboxytetramethylrhodamine) covalently attached at its $3^{\prime}$ end. A TaqMan minor-groove-binder probe consisting of a 6-carboxyfluorescein-labelled oligonucleotide at the $5^{\prime}$ end and a non-fluorescent quencher-minor-groove-binder complex ( $T_{\mathrm{m}}$ enhancer) at the $3^{\prime}$ end was used as a probe for gtfB (Kutyavin et al., 2000). Primers and probes were 
synthesized and purified by Applied Biosystems. The primers and probes used for real-time PCR are listed in Table 2; the amplicons generated with these primers were 299 and $70 \mathrm{bp}$ in length for gtfB and 16S rRNA, respectively.

Real-time RT-PCR. Total RNA was isolated from planktonic and biofilm cells using TRIZOL Reagent (Gibco-BRL) according to the manufacturer's instructions. Single-stranded cDNA was then synthesized in a reaction mixture containing 1.25 U MultiScribe Reverse Transcriptase $\mu \mathrm{l}^{-1}, 0.4 \mathrm{U}$ RNase Inhibitor $\mu \mathrm{l}^{-1}, 500 \mu \mathrm{M}$ of each dNTP, $200 \mathrm{nM}$ antisense primer, $1 \times$ Reverse Transcription buffer, $5.5 \mathrm{mM} \mathrm{MgCl}_{2}$ (TaqMan Reverse Transcription Reagents; Applied Biosystems) and $1 \mu \mathrm{g}$ total RNA at $48{ }^{\circ} \mathrm{C}$ for $30 \mathrm{~min}$. To check for DNA contamination, purified total RNA without reverse transcriptase served as a negative control. The resulting cDNA and negative control were amplified by using the TaqMan Universal PCR Master Mix (Applied Biosystems), which contained dNTPs with dUTP, AmpliTaq Gold DNA polymerase, Amperase UNG, optimized buffer and a passive reference dye. For each PCR, a mixture containing $5 \mathrm{ng}$ template cDNA, $1 \times$ Master Mix, $200 \mathrm{nM}$ of each sense and antisense primer and $250 \mathrm{nM}$ TaqMan probe was placed in a 96-well MicroAmp Optical Reaction Plate with Optical Caps (Applied Biosystems). Amplification and detection of the specific products were performed on an ABI PRISM 7700 Sequence Detection System (PE Biosystems) with the following cycle protocol: one cycle at $50^{\circ} \mathrm{C}$ for $2 \mathrm{~min}$ and one cycle at $95^{\circ} \mathrm{C}$ for $10 \mathrm{~min}$ followed by 60 cycles at $95^{\circ} \mathrm{C}$ for $15 \mathrm{~s}$ and $60{ }^{\circ} \mathrm{C}$ for $1 \mathrm{~min}$. The critical threshold cycle $(\mathrm{Ct})$ was defined as the cycle at which the fluorescence became detectable above the background fluorescence, and was inversely proportional to the logarithm of the initial number of template molecules. A standard curve was plotted for each primer-probe set with Ct values obtained from amplification of known quantities of cDNA. To check the linearity of the detection system, a cDNA dilution series $(1 / 10,1 / 100,1 / 1000$ and $1 / 10000)$ was amplified with primer pairs and probes so that a correlation coefficient could be calculated from the standard curve displaying $\mathrm{Ct}$ values. The standard curves were used to transform $\mathrm{Ct}$ values to the relative number of cDNA molecules. The quantity of cDNA for $g t f B$ was normalized to that of cDNA synthesized from $16 \mathrm{~S}$ rRNA. The relative amounts of RNA per cell were approximately the same for planktonic and biofilm cells (data not shown).

Statistical analysis. The variables for fluorescent intensities of GFP between biofilm and planktonic cells were assessed by using Student's $t$-test. A $P$ value of less than 0.05 was considered statistically significant.

\section{RESULTS}

\section{Construction of PgtfB::gfp fusions}

Initial attempts to construct a p15Aori plasmid harbouring the $g t f B$ promoter were unsuccessful. However, the gtfB promoter was successfully cloned into pUC19. Therefore, the promoterless $g f p$ gene was cloned into pUC19 to yield pAYG210. The isolation of a plasmid containing the gtfB promoter has been successful only in high-copy-number plasmids (pUCori or ColE1). A $193 \mathrm{bp}$ segment containing the $g t f B$ promoter was inserted $5^{\prime}$ to the promoterless $g f p$ gene in pAYG210 to generate plasmid pAYBG310. To generate a Streptococcus-E. coli shuttle vector that contained the PgtfB:: $g f p$ fusion, the PgtfB::gfp fragment $(1 \cdot 0 \mathrm{~kb})$ was

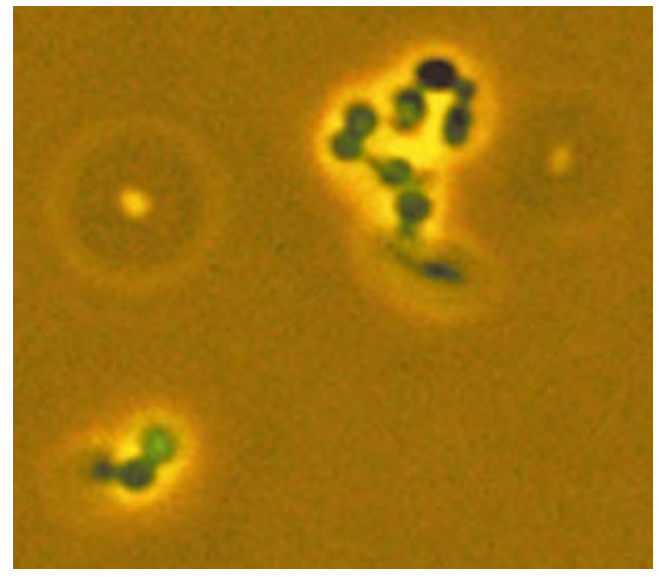

Fig. 2. Fluorescent microscopy image of $S$. mutans $854 \mathrm{~S}$ expressing GFP. The strain containing the gtfB::gfp shuttle vector is shown exhibiting varying degrees of bright-green fluorescence.

excised from pAYBG310 and cloned into the shuttle vector pDL276, which contains a Streptococcus replicon and an E. coli high-copy-number origin of replication (ori). The resulting plasmid, designated pAYBG754S, was used to transform $S$. mutans GS-5 using selection with $200 \mu \mathrm{g}$ kanamycin $\mathrm{ml}^{-1}$, but transformants were not isolated. Therefore, the erythromycin cassette from pResEmMCS10 was amplified by PCR and subcloned into pAYBG754S to generate pAYBG854S (Fig. 1). Transformation of E. coli with this shuttle vector yielded fluorescent transformants, as detected on agar plates. Transformation of S. mutans GS-5 with the plasmid extracted from $E$. coli yielded transformants at a rate of approximately 1 to $1.5 \times 10^{4}$ transformants $(\mu \mathrm{g} \text { DNA) })^{-1}$ compared with a rate of $1.5 \times 10^{4}$ transformants $(\mu \mathrm{g}$ DNA $)^{-1}$ for the same plasmid extracted from $S$. mutans 854S. To generate a chromosomal promoter fusion, the PgtfB::gfp fragment $(1 \cdot 0 \mathrm{~kb})$ was excised from pAYBG310 and cloned into the suicide vector pZ63 (Sato et al., 1991), which does not replicate in streptococci, to generate pAYBG554 (data not shown). Insertion of this plasmid into the $S$. mutans chromosome following single-cross-over integration during transformation resulted in the generation of cells with a single chromosomal copy of the PgtfB::gfp fusion.

\section{Detection of GFP-expressing S. mutans cells by fluorescence microscopy}

The visualization of GFP expressed by $S$. mutans GS-5 cells harbouring chromosomal or plasmid copies of $g f p$ was performed by fluorescence microscopy. The strains were grown anaerobically and analysed directly in CDM supplemented with $0.5 \%$ sucrose. In CDM containing $50 \mathrm{mM} \mathrm{NaCl}$, the cells harbouring both a single chromosomal copy and multiple copies of $g f p$ were not visibly fluorescent (data not shown). However, in CDM containing $50 \mathrm{mM}$ sodium phosphate buffer $(\mathrm{pH} 7 \cdot 0)$, fluorescence was readily detected in the plasmid- 
(a)

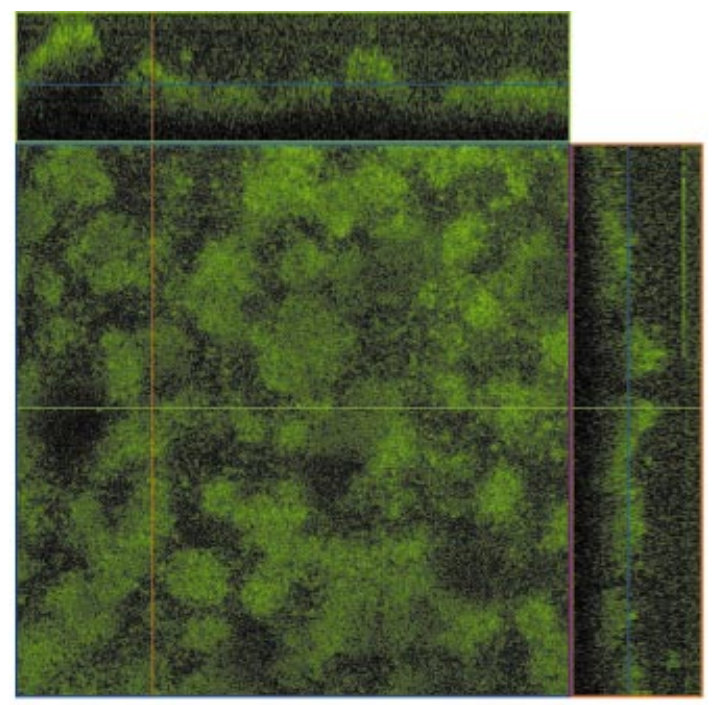

(b)

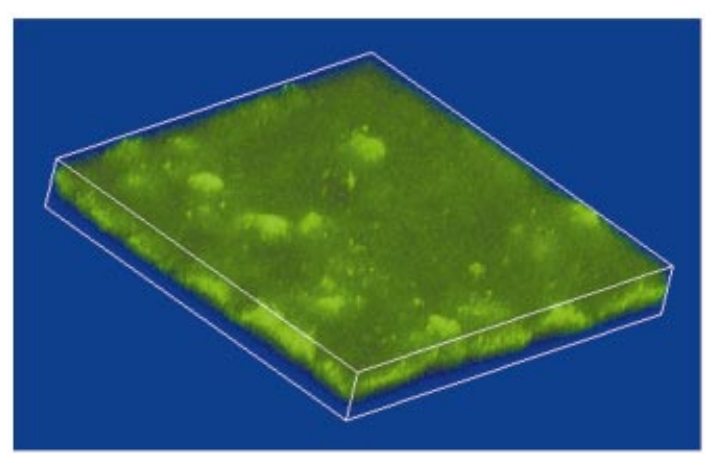

Fig. 3. CLSM images of biofilm development on a polystyrene surface by $S$. mutans 8545 carrying a plasmid which constitutively expresses GFP. (a) Topographic features of biofilm development. Sagittal section (side panels) showing large and small microcolonies linked by intercellular bridges. Dimensions of the regions displayed are $336 \times 336 \mu \mathrm{m}(x-y$ perspectives; square panel) and $336 \times 82 \mu \mathrm{m}(x-z$ perspectives; rectangular panels). (b) Three-dimensional reconstructed renderings of the biofilm. Dimensions correspond to Fig. 3(a).

containing strain (Fig. 2) but not in the strain harbouring the chromosomal copy of gfp (data not shown). These results indicated the crucial role of environmental $\mathrm{pH}$ on the detection of GFP fluorescence, as noted by Hansen et al. (2001).

\section{CLSM of GFP-expressing S. mutans cells and biofilm morphology}

The biofilm formed by the GFP-expressing $S$. mutans $854 \mathrm{~S}$ cells that bound to the polystyrene surface of the wells after $24 \mathrm{~h}$ cultivation in CDM supplemented with $0.5 \%$ sucrose is shown in Fig. 3. After $24 \mathrm{~h}$ incubation, the $S$. mutans $854 S$ cells formed several very large amorphous microcolonies and many small microcolonies were interspersed across the biofilm (Fig. 3a). The micrographs of single sections indicated an increase of
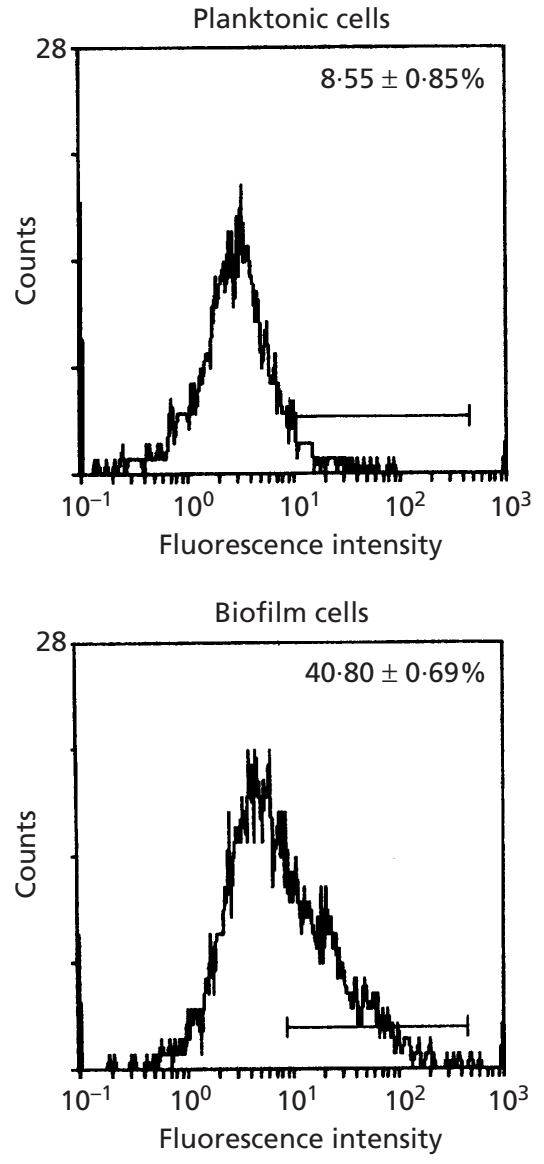

Fig. 4. Comparison of flow cytometry analysis of $g \mathrm{fp}$ expression by $S$. mutans gtfB promoters in planktonic and biofilm cells. Flow cytometry histograms of $S$. mutans $854 \mathrm{~S}$ were analysed. Bars indicate regions of positive fluorescence; the percentage of positive cells is indicated in both panels. Results are representative of four experiments.

fluorescence in the biofilm as a function of sucrose, as did the side panels ( $z x$ and $z y$ scans) on the entire stack of images. Vertical gradients of fluorescence through the biofilm were also observed (Fig. 3b).

\section{Flow cytometry analysis of GFP-expressing S. mutans cells}

GFP expression by $S$. mutans $854 S$ was also examined in harvested planktonic (S. mutans $854 \mathrm{~S}-\mathrm{P}$ ) cells and in sessile (S. mutans 854 S-B) cells attached to the polystyrene plates, by using flow cytometry. Planktonic and sessile cultures of untransformed wild-type $S$. mutans GS-5 cells served as negative controls. Cells of $S$. mutans strains 854 S-P, 854 S-B and GS-5 were subjected to flow cytometry after $24 \mathrm{~h}$ anaerobic growth in CDM supplemented with $0.5 \%$ sucrose. After $24 \mathrm{~h}$ cultivation, $8.55 \pm 0.85 \%$ (mean \pm SEM, $n=4$ ) of the S. mutans 854 S$\mathrm{P}$ cells were found in the regions of positive fluorescence (Fig. 4). In contrast, 40.80 $\pm 0.69 \%$ (mean \pm SEM, $n=4$ ) of the $S$. mutans 854 S-B cells were found in the same regions $(P<0 \cdot 01$; Fig. 4). Therefore, these results indi- 


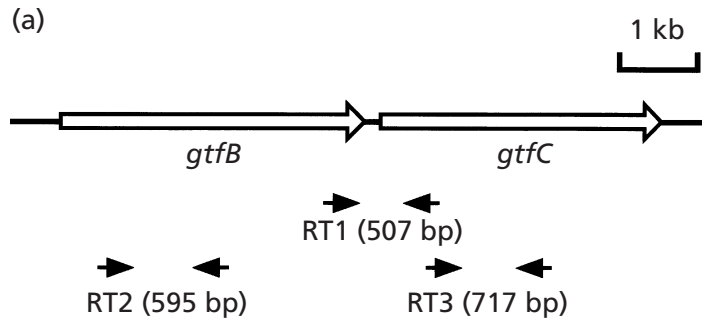

(b)

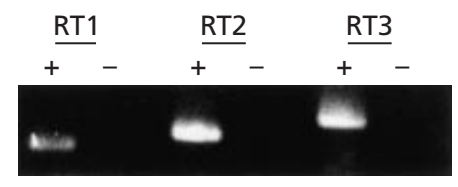

Fig. 5. Genetic organization of the gtfBC gene region and RTPCR analysis of mRNA transcripts. (a) The orientations of the gtfB and gtfC genes are indicated by open arrows. The solid arrows indicate the primers used for RT-PCR. (b) Gel electrophoresis of amplified DNA fragments. Lanes: RT1, amplification with primer pair RT1F/RT1R; RT2, amplification with primer pair RT2F/RT2R; RT3, amplification with primer pair RT3F/RT3R. +, Standard amplifications with RT-PCR; -, negative control reactions without reverse transcription.

cated an almost five-fold increase in $g t f B$ expression in the biofilm cells relative to the planktonic cells. To check the effects of different sugars on $g t f B$ regulation, the GFP expression levels of $S$. mutans $854 \mathrm{~S}$ cells grown in CDM supplemented with $0.5 \%$ sucrose, glucose or fructose were analysed by fluorescence-activated cell sorting. After $24 \mathrm{~h}$ anaerobic growth, there were no significant differences in $g t f B$ expression between planktonic cells grown in the presence of the three sugars (data not shown). These results suggested that increased $g t f B$ expression in the sessile cells relative to the planktonic cells of strain GS-5 was regulated by the environment of the biofilm.

\section{Evidence for transcription of the gtfBC locus in $S$. mutans}

We performed RT-PCR analysis to identify the transcripts of the $g t f B$ and $g t f C$ genes of $S$. mutans. A pair of oligonucleotide primers spanning the borders of $g t f B-$ gtfC (RT1F/RT1R; Table 2) was designed. In addition to RT1F/RT1R, two pairs of primers were designed to amplify $g t f B(\mathrm{RT} 2 \mathrm{~F} / \mathrm{RT} 2 \mathrm{R})$ and $g t f C$ (RT3F/RT3R) as controls (Table 2). Fig. 5(b) shows the $507 \mathrm{bp}$ PCR product amplified with the RT1F/RT1R primer pair. No PCR products were observed from total RNA preparations that had not been reverse transcribed first (Fig. 5b), indicating that the RT-PCR products were not derived from contaminating chromosomal DNA.

\section{Quantification of gtfB transcription in planktonic and biofilm cells}

Real-time RT-PCR (TaqMan) assays were employed to examine the relative abundance of $g t f B$-bearing transcripts in planktonic and biofilm cells. The test samples
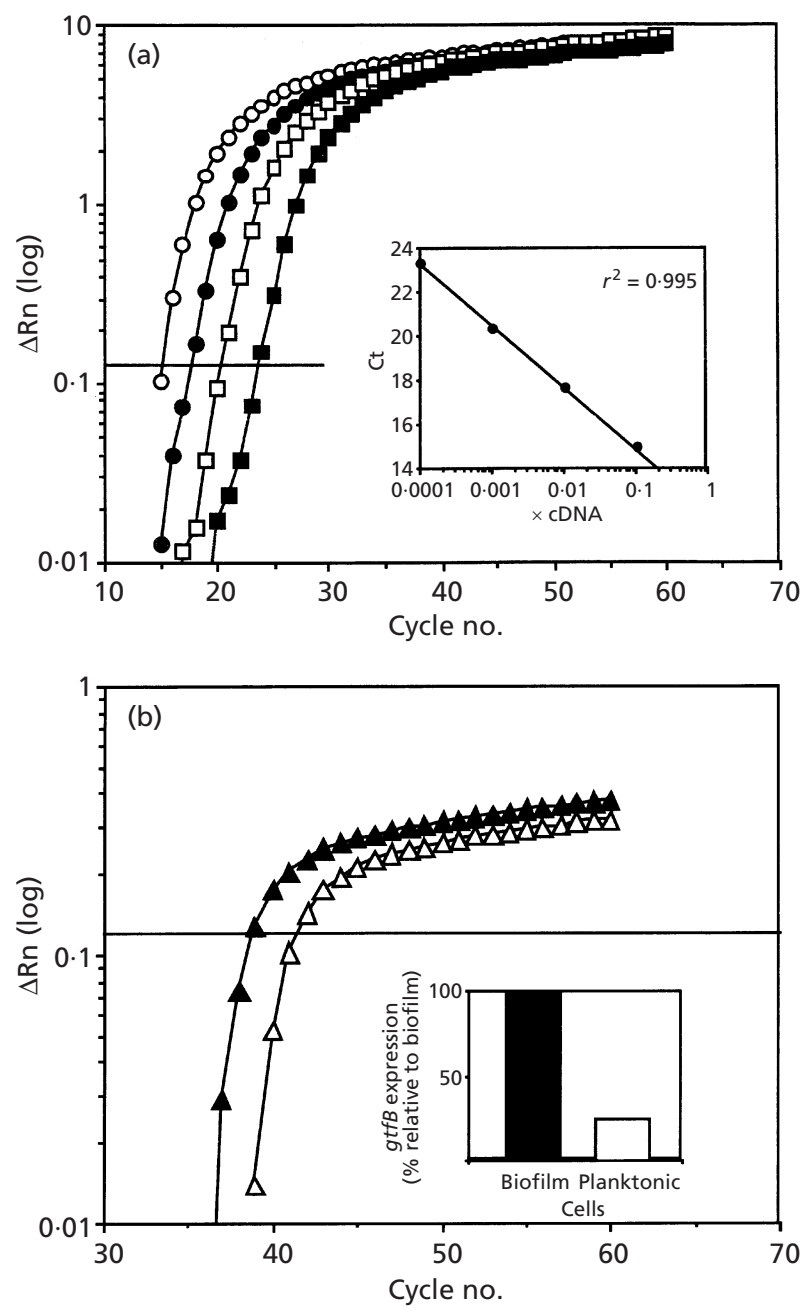

Fig. 6. Real-time quantitative RT-PCR analysis of S. mutans gtfB expression in biofilm and planktonic cells. (a) Amplification plot of S. mutans cDNA. Serial dilutions of cDNA from S. mutans $16 \mathrm{~S}$ rRNA were used as templates for real-time PCR. The horizontal bar indicates the threshold fluorescence $(T=0 \cdot 116)$. A standard curve was generated from the amplification plot $\left(r^{2}=0.995\right)$. $O$, $0.1 \times$ CDNA; $\quad, 0.01 \times$ CDNA; $\square, 0.001 \times$ CDNA;, $0.0001 \times$ cDNA. (b) Amplification plot and relative quantities of CDNA from $S$. mutans gtfB in biofilm and planktonic cells, as assessed by TaqMan assays. The amount of gtfB cDNA was determined and normalized to the amount of CDNA of 165 rRNA following reverse transcription of total RNA isolated from biofilm and planktonic cells. The mean $\mathrm{Ct}$ value of the gtfBspecific cDNA from planktonic cells was $41.049 \pm 0.521$ (mean \pm SEM, $n=4)$ and that of the CDNA from biofilm cells was $38.933 \pm 0.375$ (mean \pm SEM, $n=4$ ). The horizontal bar indicates the threshold fluorescence, as in (a). $\triangle$, Planktonic cells; $\boldsymbol{\Delta}$, biofilm cells. The results shown are representative of those obtained from two independently isolated RNA preparations.

were cDNA-primed with an antisense primer, and 10fold serial dilutions of cDNA from $16 \mathrm{~S}$ rRNA were employed to generate the standard curves. Kinetic curves were defined by a $\mathrm{Ct}$ value that marked the fractional cycle number during the exponential phase at which the fluorescence of a given sample became significantly different from the baseline signal. Kinetic curves are 
shown for four concentrations of cDNA (Fig. 6a). The linearity of $g t f B$ transcription was exactly the same for the planktonic and the biofilm cells (data not shown). For each cDNA template concentration, a single PCR product of the expected size for primer pair gtfBF241/gtfB-R539 was detected by gel electrophoresis (data not shown). The gtfB mRNA in the planktonicand biofilm-cell samples was quantified by measuring the $\mathrm{Ct}$ and by using calibration curves obtained during the same experiments to determine the quantity of target message. The results shown here are representative of four independent experiments. The same linearity of transcription was observed when the template was gtfBspecific cDNA from either planktonic or biofilm cells (data not shown). The mean Ct value of the cDNA from planktonic cells was $41.049 \pm 0.521$ (mean \pm SEM, $n=4$ ) and that of the cDNA from biofilm cells was $38.933 \pm 0.375$ (mean \pm SEM, $n=4$ ). These experiments revealed that the level of $g t f B$ mRNA expressed in biofilm cells was approximately four-fold higher than that expressed in planktonic cells (Fig. 6b).

\section{DISCUSSION}

Despite intensive investigations into GTF-encoding genes, the regulation of the expression of these genes has not been thoroughly defined. This is due primarily to the relatively large size of the $g t f$ gene products and the high nucleotide sequence similarity of the GTF-encoding genes; both of these factors have made it difficult to perform transcriptional analysis on these genes. If the role of $g t f B$ in $S$. mutans biofilm formation is to be defined, detailed transcriptional analysis of $g t f B$ is necessary; hence, we initially investigated the feasibility of utilizing $g t f B:: g f p$ reporter fusions in such studies. Consequently, the work detailed here represents the first report of GFP expression in $S$. mutans using an endogenous promoter to drive $g f p$ transcription.

PgtfB::gfp was isolated on high-copy-number plasmids (pUCori or ColE1), but not on a medium-copy-number plasmid (p15Aori) (Y. Nakano, personal communication). Interestingly, the copy number of the plasmid harbouring the PgtfB fragment was significantly decreased when compared with that of the original plasmid (data not shown). Therefore, pDL276, a high-copynumber Streptococcus-E. coli shuttle vector, was selected for subcloning the PgtfB::gfp fusion. Following transformation of pAYBG854S (pDL276 harbouring PgtfB: : gfp) into E. coli DH5 $\alpha$, GFP expression driven by the $S$. mutans PgtfB was active in E. coli on agar plates, as revealed by the green fluorescent colonies of the transformants. However, the fluorescence from the $S$. mutans transformants that expressed GFP was not detected on agar plates. In addition to this, the transformation efficiency of $S$. mutans GS-5 with pAYBG854S extracted from E. coli was quite low. However, the transformation rate of $S$. mutans GS-5 with pAYBG854S isolated from $S$. mutans 854 S was relatively high. This suggested that restriction-modification systems in E. coli may be responsible for the differences seen in transformation efficiency between pAYBG845S isolated from E. coli and the same plasmid isolated from S. mutans 854S.

For visualization of $S$. mutans 854 S cells, CLSM analysis was performed. S. mutans harbouring a single chromosomal copy of $g f p$ did not display visible fluorescence, but transformants harbouring multiple copies of $g f p$ were fluorescent. A study by Acebo et al. (2000) reported that fluorescence could be detected from Streptococcus pneumoniae cells that harboured a single chromosomal copy of $g f p$ by fluorescence microscopy. However, the gfp copy number may be one of several important factors affecting the expression of a target promoter. For example, the differences in fluorescence intensity may be influenced by variations in oxygen intake by the bacteria or by other factors (Hansen et al., 2001).

CLSM was also carried out on $S$. mutans biofilms attached to the wells of polystyrene plates. The advantage of using CLSM in biofilm studies is due to the enhanced ability this technique affords for observing and analysing fresh, undisturbed materials in real time (Lawrence \& Neu, 1999). From the CLSM images of the $S$. mutans biofilms, it was observed that $g t f B$ expression was enhanced on the surfaces of the biofilms; vertical gradients of fluorescence were also observed in the biofilms. In previously utilized flowcell systems, rapid oxygen distribution in Streptococcus gordonii biofilms has been confirmed (Hansen et al., 2001). Our results suggest that the oxygen supply may be a limiting factor in the deeper layers of the artificial biofilm. Moreover, the fluorescence pattern of the $S$. mutans biofilm (as seen in Fig. 3a) could be due either to microcolonies where $g t f B$ expression is enhanced or to gaps between microcolonies where aeration is better. In the process of bacterial biofilm formation, bacterial species migrate over the colonized surface to form microcolonies and, subsequently, produce an exopolysaccharide matrix to form and support the biofilm (Watnick \& Kolter, 2000). Thus, our findings, which suggest enhanced $g t f B$ expression in microcolonies, are consistent with this general model for biofilm formation.

Based on the initial experiments detailed here, we have examined $g t f B$ expression in planktonic and biofilm cells using two distinct approaches. In earlier work, several groups examined gt $f B C$ expression using various reporter systems with a single chromosomal copy of the reporter gene (Burne et al., 1997; Hudson \& Curtiss, 1990). It is important to consider several aspects of the promoter-reporter system when analysing the expression of the $S$. mutans gtfB and gtfC genes. In the present study, we employed GFP expression and flow cytometry to detect the expression of $g t f B$ in $S$. mutans biofilms. At first, to eliminate the effects of differences in $g f p$ copy number, we compared the ratios of plasmid to chromosomal DNA in biofilm and planktonic cells and found these to be quite similar (data not shown). The fluorescent intensity of the biofilm cells was enhanced compared with that of the planktonic cells. A study by 
Hudson \& Curtiss (1990) reported that the chloramphenicol acetyltransferase activity of $S$. mutans cells directed by the $g t f B C$ operon promoter was approximately two-fold higher in cells bound to saliva-coated beads than in unbound cells. To date, almost all of the transcription analyses done involving the $g t f B$ and $g t f C$ genes of $S$. mutans and reporter fusions have utilized single chromosomal copies of the reporter genes (Burne et al., 1997; Hudson \& Curtiss, 1990). However, a study by Goodman \& Gao (2000) indicated that transcription of the endogenous $g t f B$ and $g t f C$ genes was similar for both chromosomal and plasmid copies of these genes fused to reporters.

A general hypothesis concerning gt $f B C$ transcriptional regulation is that a promoter upstream of $g t f B$ initiates transcription of a polycistronic message which includes the $g t f B$ and the $g t f C$ genes (Ueda et al., 1988). However, several groups have reported that there is another promoter in the $g t f B-g t f C$ intergenic region that might allow the independent expression of gtfC (Goodman \& Gao, 2000; Smorawinska \& Kuramitsu, 1995). Due to difficulties in using Northern-blot analysis, it is reasonable to employ RT-PCR to characterize the transcripts of the $g t f B$ and $g t f C$ genes. The RT-PCR analysis of the $g t f B C$ genes performed here revealed that $g t f C$ can be transcribed together with $g t f B$ in a single polycistronic mRNA. This finding represents the first experimental verification of this hypothesis. Hence, based upon this result and previous results (Smorawinska \& Kuramitsu, 1995), it is suggested that $g t f C$ is most likely transcribed from the $g t f B$ and the $g t f C$ promoters. Therefore, it is likely that earlier studies done using $g t f B C$ reporter systems detected not only $g t f B$ expression but also $g t f C$ expression, since the reporter gene was fused downstream of gtfC (Burne et al., 1997; Goodman \& Gao, 2000; Hudson \& Curtiss, 1990). Therefore, the present results represent the first study of $g t f B$ promoter regulation in $S$. mutans biofilms.

To eliminate the effects of $g t f C$ expression from our results, we also performed real-time RT-PCR (TaqMan) analysis for only gtfB expression in planktonic and biofilm cells. The TaqMan RT-PCR assay is very sensitive and specific for obtaining quantitative information regarding transcription, and the use of this assay represents a significantly different approach for studying $g t f B$ expression relative to previous studies. Our analysis demonstrated that the rate of transcription of $g t f B$ in biofilm cells was approximately four times higher than that in planktonic cells. These results suggest $g t f B$ to be an important factor in $S$. mutans biofilm formation.

The stable expression of GFP by $S$. mutans transformants provides a useful method for studying the interaction(s) of this pathogen in biofilms. The use of CLSM in combination with the S. mutans GFP-expressing strain will make it possible to visualize bacterial behaviour in homogeneous and mixed-species biofilms. This approach should accelerate the characterization of the $S$. mutans gtfB gene as a virulence factor and contribute to our understanding of how $S$. mutans behaves in biofilms such as dental plaque.

\section{ACKNOWLEDGEMENTS}

This investigation was supported in part by NIH grant DE03258.

\section{REFERENCES}

Acebo, P., Nieto, C., Corrales, M. A., Espinosa, M. \& Lopez, P. (2000). Quantitative detection of Streptococcus pneumoniae cells harbouring single or multiple copies of the gene encoding the green fluorescent protein. Microbiology 146, 1267-1273.

Aoki, H., Shiroza, T., Hayakawa, M., Sato, S. \& Kuramitsu, H. K. (1986). Cloning of a Streptococcus mutans glucosyltransferase gene coding for insoluble glucan synthesis. Infect Immun 53, 587-594.

Brehm, J., Salmond, G. \& Minton, N. (1987). Sequence of the adenine methylase gene of the Streptococcus faecalis plasmid pAM 1 1. Nucleic Acids Res 15, 3177.

Burne, R. A., Chen, Y. Y. \& Penders, J. E. (1997). Analysis of gene expression in Streptococcus mutans in biofilms in vitro. Adv Dent Res 11, 100-109.

Chalfie, M., Tu, Y., Euskirchen, G., Ward, W. W. \& Prasher, D. C. (1994). Green fluorescent protein as a marker for gene expression. Science 263, 802-805.

Cormack, B. P., Valdivia, R. H. \& Falkow, S. (1996). FACSoptimized mutants of the green fluorescent protein (GFP). Gene 173, 33-38.

Davey, M. E. \& O'Toole, G. A. (2000). Microbial biofilms: from ecology to molecular genetics. Microbiol Mol Biol Rev 64, 847-867.

Dunny, G. M., Lee, L. N. \& LeBlanc, D. J. (1991). Improved electroporation and cloning vector system for gram-positive bacteria. Appl Environ Microbiol 57, 1194-1201.

Goodman, S. D. \& Gao, Q. (2000). Characterization of the gtfB and gtfC promoters from Streptococcus mutans GS-5. Plasmid 43, 85-98.

Hanada, N. \& Kuramitsu, H. K. (1988). Isolation and characterization of the Streptococcus mutans gtfC gene, coding for synthesis of both soluble and insoluble glucans. Infect Immun 56, 1999-2005.

Hanada, N. \& Kuramitsu, H. K. (1989). Isolation and characterization of the Streptococcus mutans gtfD gene, coding for primer-dependent soluble glucan synthesis. Infect Immun 57, 2079-2085.

Hansen, M. C., Palmer, R. J., Jr, Udsen, C., White, D. C. \& Molin, S. (2001). Assessment of GFP fluorescence in cells of Streptococcus gordonii under conditions of low $\mathrm{pH}$ and low oxygen concentration. Microbiology 147, 1383-1391.

Hudson, M. C. \& Curtiss, R., 3rd (1990). Regulation of expression of Streptococcus mutans genes important to virulence. Infect Immun 58, 464-470.

Kutyavin, I. V., Afonina, I. A., Mills, A. \& 11 other authors (2000). 3'-Minor groove binder-DNA probes increase sequence specificity at PCR extension temperatures. Nucleic Acids Res 28, 655-661.

Lawrence, J. R. \& Neu, T. R. (1999). Confocal laser scanning microscopy for analysis of microbial biofilms. Methods Enzymol 310, 131-144. 
Loesche, W. J. (1986). Role of Streptococcus mutans in human dental decay. Microbiol Rev 50, 353-380.

Perry, D., Wondrack, L. M. \& Kuramitsu, H. K. (1983). Genetic transformation of putative cariogenic properties in Streptococcus mutans. Infect Immun 41, 722-727.

Prasher, D. C., Eckenrode, V. K., Ward, W. W., Prendergast, F. G. \& Cormier, M. J. (1992). Primary structure of the Aequorea victoria green-fluorescent protein. Gene 111, 229-233.

Sambrook, J., Fritsch, E. F. \& Maniatis, T. (1989). Molecular Cloning: a Laboratory Manual, 2nd edn. Cold Spring Harbor, NY : Cold Spring Harbor Laboratory.

Sato, Y., Yamamoto, Y., Suzuki, R., Kizaki, H. \& Kuramitsu, H. K. (1991). Construction of $s c r A:: l a c Z$ gene fusions to investigate regulation of the sucrose PTS of Streptococcus mutans. FEMS Microbiol Lett 63, 339-345.

Shiroza, T. \& Kuramitsu, H. K. (1993). Construction of a model secretion system for oral streptococci. Infect Immun 61, $3745-3755$.
Skillman, L. C., Sutherland, I. W., Jones, M. V. \& Goulsbra, A. (1998). Green fluorescent protein as a novel species-specific marker in enteric dual-species biofilms. Microbiology 144, 2095-2101.

Smorawinska, M. \& Kuramitsu, H. K. (1995). Primer extension analysis of Streptococcus mutans promoter structures. Oral Microbiol Immunol 10, 188-192.

Ueda, S., Shiroza, T. \& Kuramitsu, H. K. (1988). Sequence analysis of the gtfC gene from Streptococcus mutans GS-5. Gene 69, 101-109.

Watnick, P. \& Kolter, R. (2000). Biofilm, city of microbes. J Bacteriol 182, 2675-2679.

Yamashita, Y., Bowen, W. H., Burne, R. A. \& Kuramitsu, H. K. (1993). Role of the Streptococcus mutans gtf genes in caries induction in the specific-pathogen-free rat model. Infect Immun 61, 3811-3817.

Received 30 April 2002; revised 12 June 2002; accepted 26 July 2002 